\title{
Research of the Books Localization Algorithm in Library Intelligent Bookshelf System Based on RFID
}

\author{
Yuanzhong Shu ${ }^{\mathrm{a}}$, Yannan Su ${ }^{\mathrm{b}}$, Xiaohong Peng and Mengzhe Mei \\ School of Information Engineering \\ Nanchang Hangkong University \\ Nanchang, China, 330063 \\ a20669237@qq.com, bynllcj@sina.com
}

\begin{abstract}
Under the currently trend of the library collection quantity increasingly, the book disordered problem became more common which caused the reader too hard to find a book that they want. To solve these problems, this paper design the accurate location algorithm based on RFID intelligent bookshelf, and set the algorithm model parameters. Through the simulation analysis, the location algorithm improves the positioning accuracy effectively, lower the system cost complexity, improve the borrowing efficiency of the readers.
\end{abstract}

Keywords-RFID technology;indoor location; received signal strength;reference tag;model parameter

\section{INTRODUCTION}

Because of the great amount of library collections, to find a book reader need is a not easy thing on a large number of books in the literature. Owing to the uncertainty of the position of books, a book was carried away by the readers after reading other shelf of bookshelf or other bookshelves, the location information of books provided by management system database may no longer be accurate, readers wasting time and energy to find books. To solve these problems, scholars introduce Radio Frequency Identification (RFID) technology to design intelligent bookshelf, through installing RFID reader on the bookshelf. Jing Xin and Zhang Jing[1] designed application framework model of intelligent bookshelf based on the RFID technology, realizing real-time inventory of books. But the current intelligent bookshelf positioning books only by increasing hardware device, result in system cost is high, so in this paper the indoor location algorithm model is set up to positioning books. Sun Yu, Zhiping Fan[3] and others put forward a nearest neighbor algorithm and data fusion location algorithm based on error of multistage processing on the basis of LANDMARC system localization algorithm. But the current indoor location algorithm complexity is too high to suit the special environment of library, and positioning accuracy needs to improve.

\section{RFID INTELLIGENT BOOKSHELF AND INDOOR LOCATION TECHNOLOGY}

\section{A. intelligent bookshelf system}

Intelligent bookshelf is the equipment scanning and collecting books information by installed RFID reader and antenna in library bookshelf. It can effectively improve the efficiency of the reader's borrowing and reduce the labor intensity of librarians.

This paper design the intelligent bookshelf from two aspects of hardware and software to achieve the requirement of real-time positioning books, based on special environment of library. The hardware architecture diagram of intelligent bookshelf is shown in Figure 1.

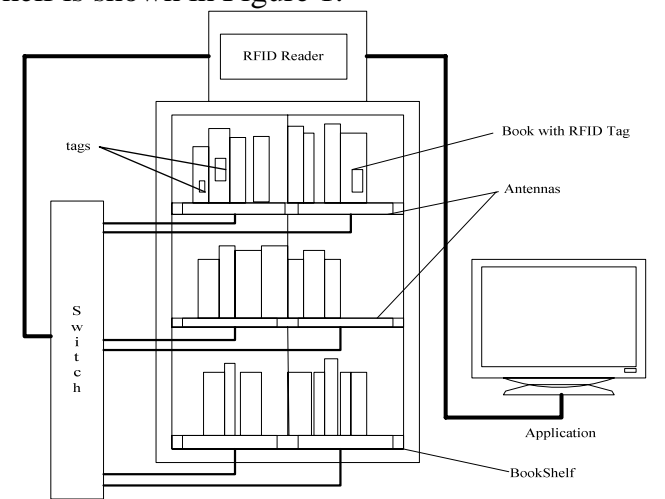

Figure 1. Hardware Architecture Diagram of Intelligent Bookshelf

As shown in graph 1, intelligent bookshelf is made up of five main parts: bookshelf, antenna group, antenna group switch group, RFID reader and data processing center.

Based on the hardware architecture, the software architecture of intelligent bookshelf system had been designed. The software architecture of intelligent bookshelf system is shown in Figure 2.

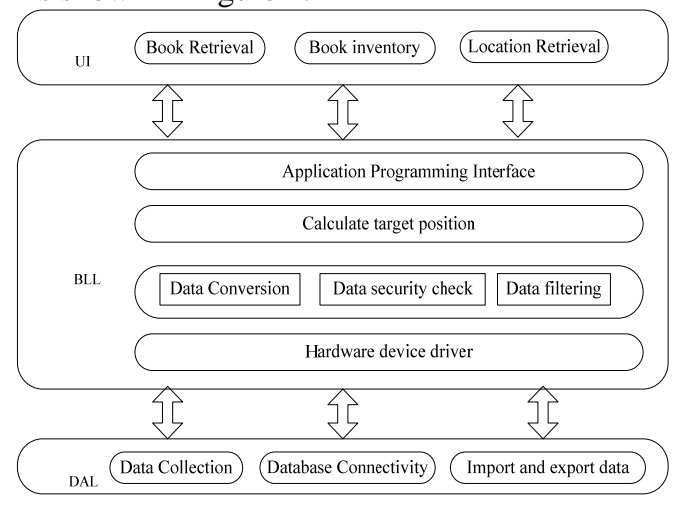

Figure 2. Software Architecture of Intelligent Bookshelf System 
Software architecture can divide into three layers from top to bottom: User Interface(UI), Business Logic Layer (BLL) and Data Access Layer (DAL). Three layers call from top to bottom to achieve the goal "high cohesion and low coupling"[4]. Thereinto books location algorithm is encapsulated on Business Logic Layer, for User Interface calling.

\section{B. Indoor location algorithm}

There are two kinds of target location-indoor and outdoor. In the field of outdoor location, satellite positioning technology mature to promote the rapid development of GPS technology [5]. This technology is mainly carried out on the large moving target location, but for the special environment of library which has complex structure and many shelters, the RFID technology breakthrough the limitations of GPS in the field of indoor location to realize indoor small target location.

At present indoor location algorithm mainly have three: time of arrival location algorithm, angle of arrival location algorithm and received signal strength location algorithm.

Time of arrival (TOA) location algorithm ranging by using moving targets and measuring propagation time of wireless signal[7]. The principle diagram of the algorithm is shown in Figure 3.
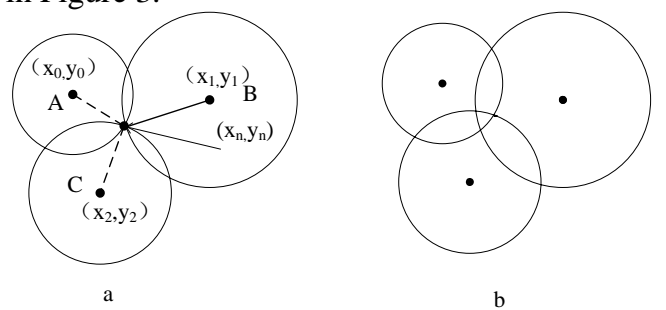

Figure 3. Principle Diagram of Algorithm

TOA algorithm requires highly synchronization of transmitter-receiver set. If positional accuracy is improved, system will introduce other algorithm to compensate position. In this way, the cost and complex of algorithm will increase, so TOA algorithm is not suitable for intelligent bookshelf system location.

Angle of arrival (AOA) location algorithm measures radio frequency identification signal transmitted by moving target through the antenna array, and receiving device measures the incident angle of electromagnetic ware, forming a location line from launcher to receiver. On this account, we can determine the direction of moving target's location, to positioning based on signal's angle of arrival[8]. The principle diagram of the algorithm is shown in Figure 4.

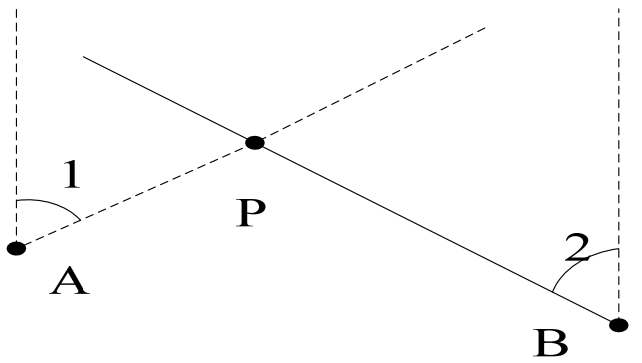

Figure 4. Principle Diagram of Algorithm

AOA location algorithm has the advantage that only need two reference points can determine the target location so that the blur positional phenomena that motion path has multiple points of intersection will not appear. But the AOA location accuracy is affected seriously by the environmental multipath effect . In order to measure accurately the incident angle, we need to improve receiver antenna and to install the directional antenna array, so AOA algorithm is not suitable for intelligent bookshelf system location either.

Received signal strength (RSS) algorithm obtains label response signal power value, that is signal strength value, through transmission power by reader, to represent the distance between label and reader and to calculate the coordinate of position target ${ }^{[9]}$. The schematic diagram of received signal strength algorithm is shown in Figure 5.

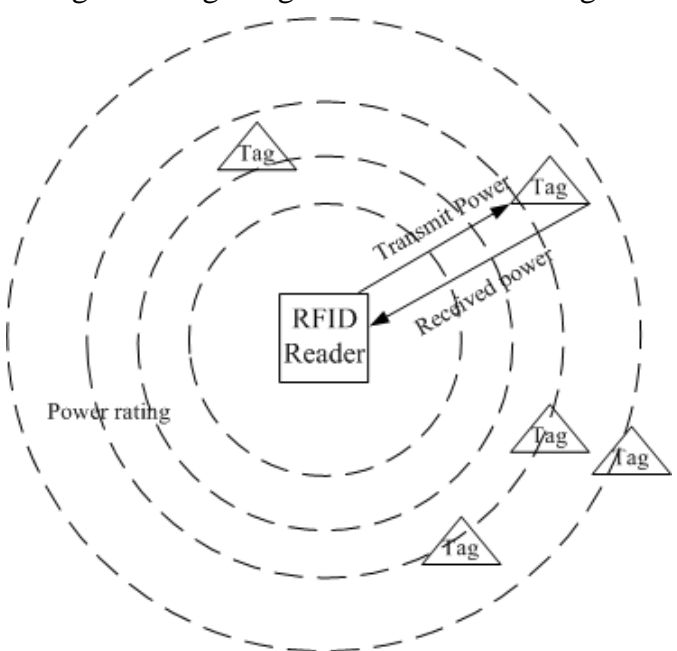

Figure 5. Schematic diagram of received signal strength algorithm

LANDMARC system is an indoor location identification system based on the signal strength of active RFID electronic label. Its research introduced the concept of reference label. Using reference label as the system positioning reference point for assistant position, system estimates the distance by obtaining the signal strength value of reference label and undetermined label, thus real-time forecasts the location of undetermined object ${ }^{[10]}$. 


\section{INTELLIGENT BOOKSHELF LOCATION ALGORITHM MODEL}

\section{A. The establishment of the location algorithm model}

Location algorithm based on received signal strength in RFID indoor location has a strong advantage, so intelligent bookshelf system uses additional reference labels to obtain location information, reaching the higher locating accuracy without increase of the number of readers. Reference labels provide the function of location identification, so that supplying reference location information for system algorithm. It is suitable to achieve the function of real time positioning books in a library environment. This paper will use the passive label as reference label and books' label.

Normally the library bookshelves are almost matrix arrangement. According to the idea of LANDMARC system, this paper uses pattern matching method based on received signal strength (RSS) to positioning books, that is the nearest neighbors algorithm ${ }^{[11]}$. Intelligent bookshelf designed in this paper need to be installed a reader in each shelf, thus every intelligent bookshelf is an independent real-time location system unit. In continuous working mode, reader detects once every 30 seconds. Assuming that reader's number is 1 , system database recorded the signal strength vector $\mathrm{P}$ of reference label in advance. Qi expresses the ith signal strength vector of undetermined label, therefore the distance between reference label and undetermined label can represent as:

$$
E_{\mathrm{j}}=\left|\mathrm{P}_{\mathrm{j}}-\mathrm{Q}_{\mathrm{i}}\right|, \mathrm{j} \in(1, \mathrm{~m})
$$

The smaller $\mathrm{E}$ express the closer the distance between undetermined label and reference label. The coordinate of known undetermined label is $(\mathrm{x}, \mathrm{y})$, so its coordinate expression is:

$$
(\mathrm{x}, \mathrm{y})=\sum_{\mathrm{i}=1}^{\mathrm{k}} W_{\mathrm{i}}\left(\mathrm{x}_{\mathrm{i}}, \mathrm{y}_{\mathrm{i}}\right)
$$

Thereinto $\left(x_{i}, y_{i}\right)$ express the coordinates of reference label recorded in system database; $k$ express the number of setting neighbor label, also is $\mathrm{n}$ reference labels of the smallest E value; Wi express the weight of the ith neighbor label.

To determine weight need to meet two conditions: sum of weight need to meet $\mathrm{w}_{1}+\mathrm{w}_{2}+\cdots+\mathrm{w}_{\mathrm{n}}=1$; weight is monotone decreasing function ${ }^{[12]}$. So the weight in accordance with conditions has three followings:

The weight of the first order:

$$
W_{i}=\frac{\frac{1}{E_{i}}}{\sum_{i=1}^{k} \frac{1}{E_{i}}}
$$

The weight of the second order:

$$
W_{i}=\frac{\frac{1}{E_{i}^{2}}}{\sum_{i=1}^{k} \frac{1}{E_{i}^{2}}}
$$

The weight of logarithmic:

$$
W_{i}=\frac{\frac{1}{\log E_{i}}}{\sum_{i=1}^{k} \frac{1}{\log E_{i}}}
$$

Define a variable used to describe the position of undetermined label and signal strength, and calculate proximity of reference label of smallest E value by Euclidean distance formula, that is single label dependence $\xi$.

$$
\xi=\left(W_{i}\right)_{\max }
$$

With the same characteristic distance $\mathrm{E}_{\mathrm{i}}$ of undetermined label, the greater label dependence $\xi$ instructs the greater single label dependence, namely, position of undetermined label calculated is closer to the position of nearest neighbor reference label with the largest weight. A single label dependence graph under different weights setting methods is shown in Figure 6.

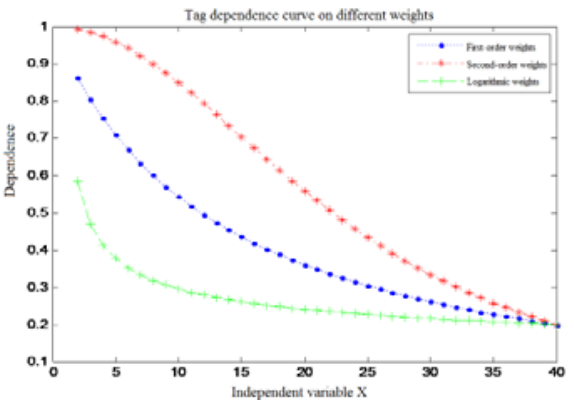

Figure 6. Dependence graph under different weights setting methods

As shown in figure, a single label dependence is the highest using the setting method of weight of the second order and is the lowest using the setting method of weight of logarithmic. So the weight of formula (2) should be weight of the second order.

\section{B. The parameter settings of the location algorithm model}

1) Distribution of reference label: To make parameters assessing the positional accuracy be average position error, the expression can be expressed as:

$$
\mathrm{MEE}=\frac{1}{\mathrm{M}} \sum^{M} \sqrt{\left(\mathrm{x}-x_{0}\right)^{2}+\left(y-y_{0}\right)^{2}}
$$

Thereinto, the actual location coordinates of undetermined label is $(\mathrm{x} 0, \mathrm{y} 0)$. The estimate coordinates using location algorithm is $(\mathrm{x}, \mathrm{y}) . \mathrm{M}$ is the number of simulation experiments. 
Because the position of undetermined label is based on the positions of several reference labels around it, the method reference label distributing will significantly affect the accuracy of algorithm. The purpose of intelligent bookshelf system is accurately locating to the minimum cell, so we take a minimum cell as the research object, and then extend to whole bookshelf. The average error distribution figure of diffident reference labels is shown in Figure 7.

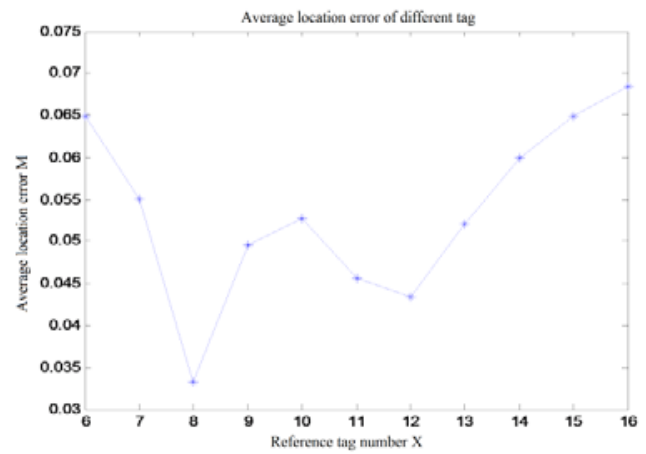

Figure 7. Error distribution figure of diffident reference

Through simulation analysis, average location error is minimum that average of 100 times location error is $0.0239 \mathrm{~m}$, when the number of reference labels are 8 . Considering each minimum cell has 8 vertices on its top and bottom surfaces.

2) The number of nearest neighbor label: The nearest neighbor label is the reference label whose characteristic distance $\mathrm{E}$ with undetermined label is minimum. The number of nearest neighbor labels will decide the number of reference labels involved in the calculation in system algorithms, significantly influencing coordinate values of undetermined target label. Considering reference labels with larger error may enhance error when location coordinates calculate weighted, we need to determine the number of nearest neighbor labels $\mathrm{k}$ in order to further improve the location accuracy of system algorithm. The error distribution figure of diffident number of nearest neighbor label is shown in Figure 8.

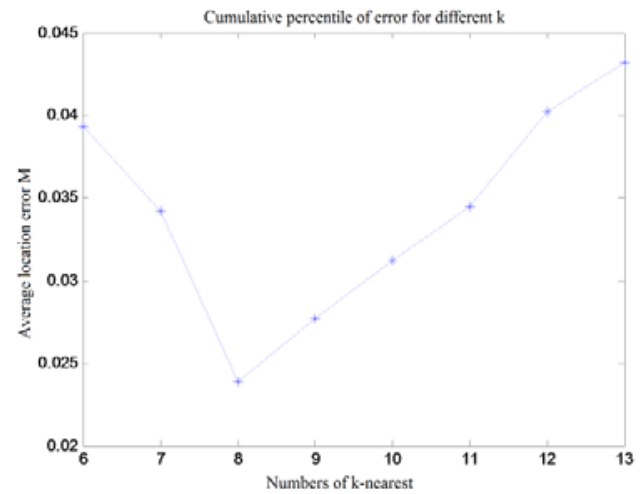

Figure 8. Error distribution figure of diffident number of nearest neighbor
From the results of simulation analysis, average location error is minimum that average of 100 times is $0.0239 \mathrm{~m}$, when the nearest neighbor label number are 8 . So we let the nearest neighbor label number $\mathrm{k}=8$.

\section{The process of intelligent bookshelf location algorithm}

Localization algorithm process is established by the intelligent bookshelf location algorithm model based on RFID technology and the conditions met by parameters.

First labels and the reader must be initialized. The nearest neighbors label number set 8 , the reader reading undetermined labels' signal strength value. Because the reference label number are $\mathrm{j}$, $\mathrm{j}$ loop calculation results, and then select a minimum of eight $E$ value, calculated undetermined label coordinates according to the weight formula. The process of intelligent bookshelf location algorithm is shown in Figure 9.

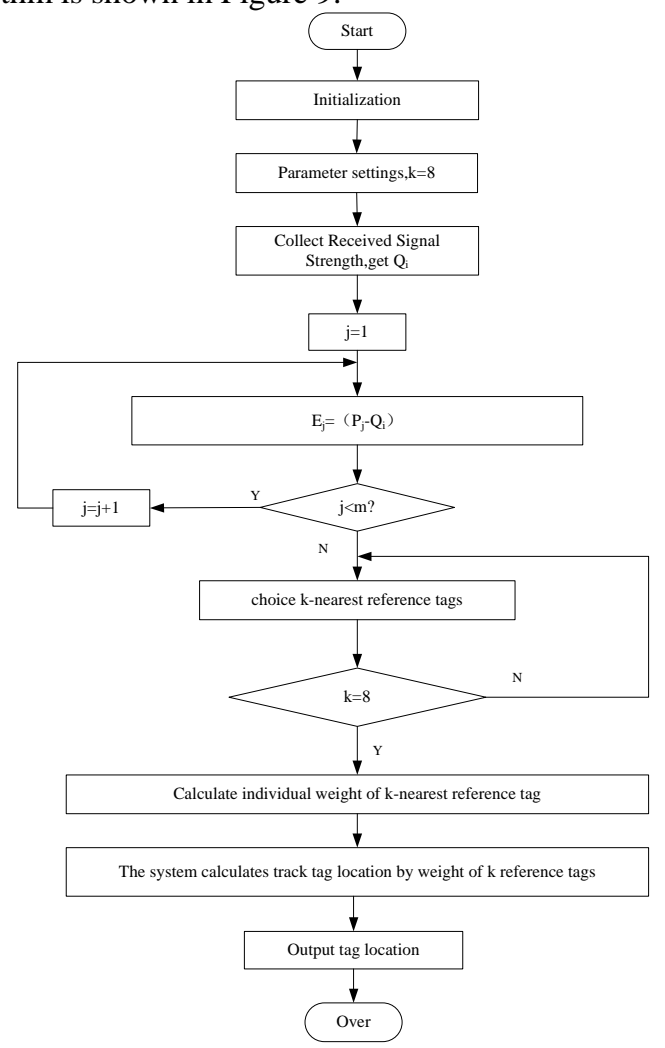

Figure 9. Process of intelligent bookshelf location algorithm

\section{ANALYSIS OF EXPERIMENTAL RESULTS}

Through the experiments we can validate the effectiveness of intelligent bookshelf location algorithm, with system configuration as described above. To locate in the four minimum adjacent cells, specification for $1.0 \mathrm{~m} * 0.5 \mathrm{~m} * 0.5 \mathrm{~m}$, of two sides of a layer of intelligent bookshelf, 8 reference label pasted respectively in eight apex angles of minimum cells, two adjacent cells joint sharing four connection labels, we can get undetermined label distribution of actual coordinates and estimated coordinates 
shown in Figure 10, without considering condition that spatial coordinate is in the vertical direction.

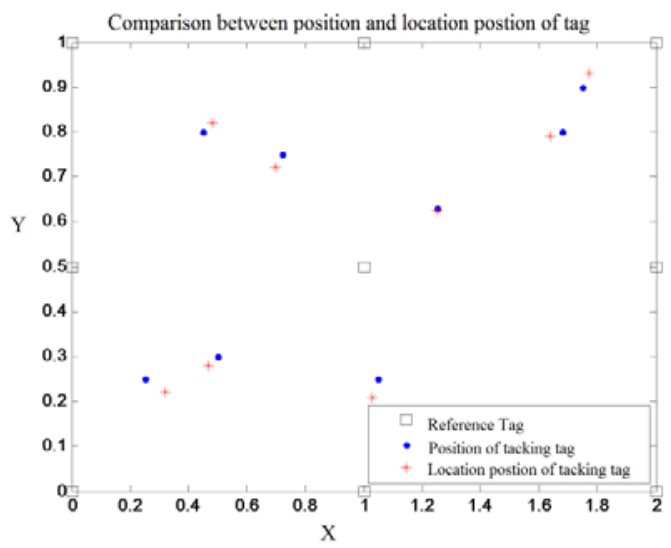

Figure 10. Distribution of actual coordinates and estimated coordinates

The average position error of undetermined label coordinates is $0.0236 \mathrm{~m}$ by calculating.So location algorithm of intelligent bookshelf gains higher accuracy, to satisfy readers' demand to locate books to minimum cell. Take the same undetermined labels' position coordinates, the average position error is $0.1416 \mathrm{~m}$ through the simulation calculation by LANDMARC algorithm. The average error is less than using LANDMARC algorithm. Error contrast figure between LANDMARC algorithm and intelligent bookshelf location algorithm is shown in Figure 11. There is the linear error ratio.

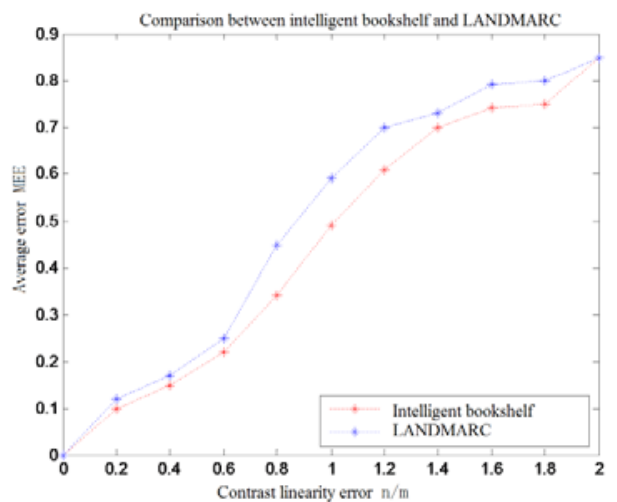

Figure 11. Error contrast figure between LANDMARC algorithm

\section{CONCLUSION}

Indoor location algorithm is a new program of locating small object, which can obtain better effect for the special environment of libraries. Library intelligent bookshelf based on RFID technology in this paper is conducive to improve management of library books efficiency, and the book location algorithm based on LANDMARC algorithm improves positional accuracy obviously. Compared to the current indoor location algorithm, complexity of the algorithm has also been reduced and the costs will be greatly reduced. Due to achieve a rapid and accurate location of books, efficiency of readers borrowing and overall service level of library will rise.

\section{REFERENCES}

[1] Zhikan Z, Dong W. Intelligent Library Bookshelf shuffling System based on RFID Technology[J]. 2013.

[2] Ng W W Y, Qiao Y S, Lin L, et al. Intelligent book positioning for library using RFID and book spine matching[C]//Machine Learning and Cybernetics (ICMLC), 2011 International Conference on. IEEE, 2011, 2: 465-470.

[3] Liu W, Ng B Q, Lim T, et al. A novel RFID and capacitive sensing based smart bookshelf[C]//Networks (ICON), 2012 18th IEEE International Conference on. IEEE, 2012: 92-97.

[4] Luo Y C, Cao J J, Qian J F. Exploration and Construction of Smart Library Based on RFID Technology[J]. Advanced Materials Research, 2013, 765: 1743-1746.

[5] Thompson B, Buehrer R M. Cooperative indoor position location using reflected estimations[C]//Wireless Conference 2011Sustainable Wireless Technologies (European Wireless), 11th European. VDE, 2011: 1-6.

[6] Saad S S, Nakad Z S. A standalone RFID indoor positioning system using passive tags[J]. Industrial Electronics, IEEE Transactions on, 2011, 58(5): 1961-1970.

[7] Bouet,Mathieu. RFID Tags: Positioning Principles and Localization Techniques[J].Wireless Days,2008,24(27):1-5.

[8] Uisheon Kim,Jeseong Koo,Jaehoon Choi.Design of a UHF band RFID Tag Antenna for Library Management System Application[J].Microwave Conference,2008,20(16):1-4.

[9] Ni L M, Liu Y, Lau Y C, et al. LANDMARC: indoor location sensing using active RFID[J]. Wireless networks, 2004, 10(6): 701-710.

[10] Lee J, El-Khatib K. A privacy-enabled architecture for an RFIDbased location monitoring system[C]//Proceedings of the 7th ACM international symposium on Mobility management and wireless access. ACM, 2009: 128-131.

[11] Daim,Terence Jerome. Relationship between RFID Readers' Output Power and Detected Transponder Distance - A Preliminary Study for 3D RFID Library Search System[J]. Innovative Technologies in Intelligent Systems and Industrial Applications2009,25(26):379-382.

[12] Zhou J, Shi J. A comprehensive multi-factor analysis on RFID localization capability[J]. Advanced Engineering Informatics, 2011, 25(1): 